\section{CRISPR genome editing}

\section{By Chris Cain, Senior Writer}

Five separate research teams have developed a Cas9 endonucleasebased method for sequence-specific genome modification that is guided by DNA-RNA base pairing, and at least two companies are commercializing the findings. ${ }^{1-6}$ Although the approach is technically more straightforward than existing methods, such as zinc finger nucleases and transcription activator-like effector nucleases, its specificity has yet to be fully determined.

At least four companies are using zinc finger nucleases (ZFNs) and transcription activator-like effector nucleases (TALENs) to engineer point mutations, deletions and insertions into the genomes of organisms ranging from plants to humans. These technologies have enabled functional genetic studies in model systems including rats, pigs and zebrafish in which it was previously difficult to make targeted knockouts.

Sangamo BioSciences Inc. has advanced ZFN-based therapeutics into the clinic.

The success of both ZFN- and TALEN-based methods hinges on designing and synthesizing complex DNA-binding domains that have a high level of target sequence specificity.

"These approaches can provide exquisite target specificity; however, protein-DNA recognition, just as with antibody-antigen specificity, is a complex interaction that is difficult to engineer from the point of view of writing it down on a piece of paper," noted Sangamo CSO and VP of research Philip Gregory.

Zinc finger motifs represent the most common and best understood DNA-binding domain in eukaryotes, although there is no single blueprint that can be followed to design a multi-zinc finger motif that will bind a given DNA sequence. Because of this, successfully designing ZFNs often requires specialized expertise.

By comparison, TALENs are relatively easy to design. The DNA specificity of TALENs derives from peptides consisting of 33-35 amino acid repeats, each of which specifically recognizes a single cognate nucleotide. By stringing together multiple peptides, a TALEN can be directed to bind to almost any target gene of interest. ${ }^{7,8}$

Since this DNA-binding code was cracked in 2009, the use of
TALENs for targeted gene knockouts in unconventional model systems has increased rapidly. However, the large size and repetitive nature of TALENs can make cloning difficult, which limits their use in some systems and makes it hard for researchers to efficiently construct the proteins in their own labs.

Thus, five separate teams of researchers set out to design a simplified genome-engineering method guided solely by DNA-RNA base pairing.

The groups built upon recent biochemical studies describing a bacterial acquired immunity-like system that relies on clustered regularly interspaced short palindromic repeats (CRISPR) to cleave unwanted exogenous DNA from phages, plasmids and other sources.

CRISPR units form the backbone of this protection system. Bacteria use CRISPR-associated (Cas) proteins to cleave foreign DNA into short fragments that are subsequently incorporated into CRISPR. These DNA fragments are then transcribed into RNA and are incorporated into a complex with other Cas proteins, including the Cas9 endonuclease. ${ }^{9}$

When foreign DNA homologous to a particular CRISPR RNACas9 complex enters the bacterial cell, the complex recognizes it and triggers its cleavage.

Last year, a team from the University of California, Berkeley and Umea University showed that the CRISPR-Cas9 system could be used to target and cleave any chosen DNA sequence in vitro. ${ }^{10}$

The logical next step was to determine whether CRISPR RNA-Cas9 complexes could be engineered to direct precise DNA cleavage in an intact genome.

The five teams took up this challenge, designing similar systems in which Cas9 was expressed in conjunction with a guide RNA containing CRISPR-specific sequences framing a region of about 20 base pairs complementary to a specific locus in the target genome.

Four teams used the approach to precisely cleave specific genomic sites and induce deletions or point mutations in human and mouse cells, ${ }^{1,2,4,6}$ whereas the fifth group demonstrated the approach's utility in zebrafish. ${ }^{3}$ One of the teams also adapted the approach to edit the genomes of two distinct bacterial species. ${ }^{5}$

In addition to targeting an individual genomic locus, some of the groups showed that multiple loci could be simultaneously targeted by a CRISPR RNA containing multiple regions of complementarity.

The approach's efficacy was comparable to that of ZFN and TALEN approaches. None of the studies provided detailed comparative data on specificity or a comprehensive genomewide specificity analysis.

The teams included researchers from the University of California, Berkeley, the Broad Institute of MIT and Harvard, the Wyss Institute for Biologically Inspired Engineering at Harvard University, Massachusetts General Hospital, The Rockefeller University, Seoul National University and Harvard Medical School. 
Results were published in two Science papers, in three Nature Biotechnology papers and in eLife.

\section{Practical advantages}

The simplicity offered by the new approach could fuel uptake and acceptance of genome-editing approaches throughout the scientific community.

"Practically, it's not only easier to design an oligonucleotide, it's a lot cheaper to synthesize. Instead of going through rounds of molecular biology in order to build a DNA-binding protein, we can just order oligos from standard suppliers," said Feng Zhang, lead investigator on one of the Science papers and a member of the Broad Institute.

In contrast to short oligonucleotides, which can be synthesized for a few dollars on average, Cellectis S.A. sells custom-engineered TALENs for about \$5,000 each, and Sigma-Aldrich Corp. prices custom ZFNs at about $\$ 6,000$ under a license from Sangamo.

Zhang plans to use the CRISPR system in his own research to study the effect of point mutations on cellular disease phenotypes. "It really allows us to scale up our ability to study these mutations. With TALENs it takes a lot of effort to build each one, but it is easy to design 50 RNAs," he said.

Jennifer Doudna, corresponding author on the eLife paper, told SciBX that the rapid development of this system by five separate teams is a testament to its real-world utility. "It's been an exciting opportunity to see it working in so many people's hands-that says a lot," she said.

Doudna is a professor of biochemistry, biophysics and structural biology at UC Berkeley and a Howard Hughes Medical Institute investigator.

Gregory predicted that uptake of CRISPR could create a knock-on effect for more established genome-editing methods. "Between zinc finger proteins and these newer methods, my guess is you will see a displacement of RNAi as the research reagent of choice for asking gene function-related questions in the future," he said.

J. Keith Joung, lead investigator on one of the Nature Biotechnology papers and associate professor of pathology at Massachusetts General Hospital, said it is important to have all the options on the table when designing experiments. "We really go on a case-by-case basis," he said. "It depends on the needs for a particular project and the limitations of the experimental system we are working in. This is why we have all three capabilities in our lab. There is no approach at this point that is necessarily one size fits all."

For example, he said, "some projects might require very precise targeting of DNA sequences, in which case we would favor the use of TALENs with their essentially limitless targeting range. Alternatively, other projects might require the use of lentiviral vectors to deliver nuclease-encoding DNA to cells, and so in those cases we would favor the use of ZFNs."

Joung has published detailed resource papers containing protocols for researchers to produce customized ZFNs and TALENs, and he maintains an open-source software package that can be used to design genomeediting experiments using all three systems. ${ }^{7,11}$ Zhang also maintains a website with tools for CRISPR and TALEN engineering.

Luciano Marraffini, assistant professor of bacteriology at Rockefeller University and a corresponding author on the bacterial genome-editing paper, acknowledged that most of the excitement is focused on eukaryotic cells but noted that there will be uses for the approach even in some bacterial systems.

"I believe that the use of CRISPR-Cas systems for bacterial mutagenesis will open the door for many high throughput studies that will certainly advance our understanding of gene networks," he said.

\section{Commercial aspirations}

At least two companies, Caribou Biosciences Inc. and ToolGen Inc., see commercial potential in the new approach.

Caribou president, CEO and cofounder Rachel Haurwitz told SciBX that the company was founded to develop and commercialize DNA and RNA manipulation tools using CRISPR-based systems. "Caribou plans to commercialize the Cas9 platform technology via internal product development and strategic partnerships with key entities in a variety of potential fields of use," she said.

Caribou was founded in October 2011 by a Berkeley team that includes Doudna, and the company is currently housed at the California Institute for Quantitative Biosciences (QB3) incubator at UC Berkeley. Doudna has filed a patent covering her work on CRISPR editing.

A second company, South Korea-based service-provider ToolGen, is planning to offer custom CRISPR-based services to researchers based on the method published by Seoul National University in Nature Biotechnology, dubbed RNA-guided endonucleases (RGENs). ToolGen provided research funding to corresponding author Jin-Soo Kim, an associate professor at the university and cofounder of the company.

Kim told SciBX that he sees a commercial opportunity in offering researchers a convenient, validated product. "We have found that not all RGENs are equally efficient, and some RGENs have no activity at all. ToolGen plans to provide high activity, validated RGENs."

He added that the company also plans to use the method to provide custom gene knockout and knock-in services in cell lines and animals. Kim has filed a patent covering his work on RGENs.

Zhang has also filed a patent application covering his CRISPR-editing work, as has George Church, corresponding author on the second Science paper and a founding core faculty member of the Wyss Institute.

Joung and Doudna noted that Church shared results from his team with them prior to publication, whereas Zhang collaborated with Marraffini.

\section{Specific questions}

The scope of the commercial and possible clinical utility of the CRISPR approach is likely to be determined by ongoing attempts to further characterize and improve specificity.

Gregory wanted to see more data detailing the off-target effects of genome modification using the CRISPR system. He noted that none of the papers measured the genomewide specificity of the approach.

"Work from Jennifer Doudna and others has shown that 12-14 bases are all that is necessary to induce Cas9 cleavage, and outside of this core region mismatches are perfectly well tolerated. To put that into perspective, any given 12-bp region would be predicted to occur by chance about 200 times in the human genome," he said.

Although at least one recent paper has shown that some ZFNs have off-target effects, ${ }^{12}$ Gregory noted that Sangamo published a wholegenome sequencing study in 2011 that showed a ZFN could correct a point mutation in a single cell and that the only change attributable to the $\mathrm{ZFN}$ action was the intended correction. ${ }^{13}$ 
Sangamo's lead therapeutic ZFN program is SB-728-T, an autologous $\mathrm{CD}^{+} \mathrm{T}$ cell therapy for patients with HIV that uses cells modified at $\mathrm{CC}$ chemokine receptor 5 (CCR5; CD195) by a ZFN.

Cellectis CSO Philippe Duchateau agreed that specificity is a key issue for CRISPR. "Only 12-14 base pairs seem to be important, and thus one can imagine that the specificity of such molecules will not be as stringent as the TALENs or zinc finger approaches," he said.

In addition to providing fee-for-service TALEN design for research labs, Cellectis is collaborating with the University College London to use its technology to engineer T cells for cancer immunotherapy.

Gregory was not hopeful about the prospect of improving the approach's specificity. "The danger with the CRISPR system is that because it is so simple, I am not convinced it can be engineered to be completely specific," said Gregory.

However, Doudna told SciBX that she was more optimistic. "The Cas9 system is one example of dozens if not hundreds of proteins with similar properties, which I suspect will be investigated very quickly by many labs. I personally think it's going to be possible to find ways to make it more selective than it is currently."

Zhang added that a better structural understanding of the Cas 9 system may provide clues to improving the approach's specificity. He noted that no Cas9-CRISPR crystal structure has been published and agreed with Doudna that studying the cleavage requirements of Cas9 homologs in other bacterial species could provide additional insight. Doudna said next steps in her lab include additional structural studies.

Joung said future work in his lab will include studying the determinants of CRISPR RNA specificity. He emphasized that the key next step to understanding the specificity of the approach is to more clearly define the rules governing CRISPR RNA-mediated cleavage of DNA. Marraffini noted that deep sequencing performed by his group has shown that some variation may be tolerated by Cas9 in certain CRISPR RNA sequences previously thought to be strictly invariant.

Doudna said that genomewide specificity analyses are ongoing in her lab and will be essential going forward.

Joung added that this system will benefit from the years of experience that have gone into developing ZFNs and TALENs. "It's important to keep in mind that ZFNs were described in '96, and we have had more than 15 years of experience optimizing and delivering them. It's been a little over three years since the TALEN code was described, and their rapid progress was because we were able to leverage what was done with the zinc fingers. With CRISPR it will be even faster."

Cain, C. SciBX 6(4); doi:10.1038/scibx.2013.77

Published online Jan. 31, 2013

\section{REFERENCES}

1. Cong, L. et al. Science; published online Jan. 3, 2013; doi:10.1126/science.1231143

Contact: Feng Zhang, Broad Institute of MIT and Harvard, Cambridge, Mass. e-mail: zhang_f@mit.edu

2. Mali, P. et al. Science; published online Jan. 3, 2013;

doi:10.1126/science. 1232033

Contact: George M. Church, Harvard Medical School, Boston, Mass.

e-mail: gchurch@genetics.med.harvard.edu

3. Hwang, W.Y. et al. Nat. Biotechnol.; published online Jan. 29, 2013; doi:10.1038/nbt.2501

Contact: J. Keith Joung, Massachusetts General Hospital, Charlestown, Mass.

e-mail: jjoung@partners.org

Contact: J.-R. Joanna Yeh, same affiliation as above

e-mail: jyeh1@partners.org

4. Jinek, M. et al. eLife; published online Jan. 29, 2013 ; doi:10.7554/eLife. 00471

Contact: Jennifer Doudna, University of California, Berkeley, Berkeley, Calif.

e-mail: doudna@berkeley.edu

5. Jiang, W. et al. Nat. Biotechnol.; published online Jan. 29, 2013; doi:10.1038/nbt.2508

Contact: Luciano A. Marraffini, The Rockefeller University, New York, N.Y.

e-mail: marraffini@rockefeller.edu

Contact: David Bikard, same affiliation as above

e-mail: dbikard@rockefeller.edu

6. Cho, S.W. et al. Nat. Biotechnol.; published online Jan. 29, 2013; doi:10.1038/nbt.2507

Contact: Jin-Soo Kim, Seoul National University, Seoul, South Korea

e-mail: jskim01@snu.ac.kr

7. Reyon, D. et al. Nat. Biotechnol. 30, 460-465 (2012)

8. Miller, J.C. et al. Nat. Biotechnol. 29, 143-148 (2011)

9. Wiedenheft, B. et al. Nature 482, 331-338 (2012)

10. Jinek, M. et al. Science 337, 816-821 (2012)

11. Maeder, M.L. et al. Mol. Cell 31, 294-301 (2008)

12. Pattanayak, V. et al. Nat. Methods 8, 765-770 (2011)

13. Yusa, K. et al. Nature 478, 391-394 (2011)

\section{COMPANIES AND INSTITUTIONS MENTIONED}

Broad Institute of MIT and Harvard, Cambridge, Mass. California Institute for Quantitative Biosciences, San Francisco, Calif.

Caribou Biosciences Inc., Berkeley, Calif.

Cellectis S.A. (Euronext:ALCLS), Paris, France

Harvard Medical School, Boston, Mass.

Howard Hughes Medical Institute, Chevy Chase, Md.

Massachusetts General Hospital, Boston, Mass.

The Rockefeller University, New York, N.Y.

Sangamo BioSciences Inc. (NASDAQ:SGMO), Richmond, Calif.

Seoul National University, Seoul, South Korea

Sigma-Aldrich Corp. (NASDAQ:SIAL), St. Louis, Mo.

ToolGen Inc., Seoul, South Korea

Umea University, Umea, Sweden

University College London, London, U.K.

University of California, Berkeley, Calif.

Wyss Institute for Biologically Inspired Engineering at

Harvard University, Cambridge, Mass. 